\title{
Yoga en España. Proceso de regulación profesional
}

\author{
Yoga in Spain. \\ Professional Regulation Process
}

\author{
María Albert Rodrigo \\ Universitat de Valencia
}

\section{RESUMEN}

El desarrollo de nuevas espiritualidades va acompañado de emergentes movimientos físicopsico-espirituales que han tenido un claro impacto en ámbitos distintos, pero especialmente en el de la salud y el bienestar debido a su intensa proliferación. Cada día es más frecuente encontrar enfermeros/as que ofrecen yoga a sus pacientes, médicos/as que recomiendan mindfulnes (meditación), etc. El uso de las llamadas Medicinas Alternativas y Complementarias (MAC) ocupa un lugar cada vez más destacado en nuestra sociedad. Se trata de una realidad ya bien visible en los conocidos centros, escuelas, consultas particulares, etc. de terapias alternativas. La proliferación de todos estos espacios terapéuticos camina de la mano de la necesidad de una regulación institucional tanto para el reconocimiento de profesionales que operan en este campo como para su formación. España, hoy es protagonista de un importante avance en materia jurídica en la regulación de la Instrucción en Yoga a raíz de los Reales Decretos publicados en los últimos años, así como de los procesos de acreditación y de habilitación de los profesores de yoga en el caso catalán. En este artículo vamos a ver como se está realizando dicho proceso, mostrando los interrogantes que se han generado, los acuerdos y las discrepancias entre el sector y la administración, así como dentro del propio sector.

Palabras clave: Yoga; Medicinas alternativas y complementarias (MAC); Regulación profesional; New Age.

\section{SUMMARY}

New approaches to spirituality go hand in hand with emerging physical, psychological and spiritual movements that have had a clear impact on many areas, and in particular on health and well-being. It is increasingly common to find nurses teaching yoga or doctors prescribing mindfulness. Use of socalled Complementary and Alternative Medicine (CAM) is becoming mainstream. Health centres, medical practices and even schools are offering a growing number of alternative therapies, resulting in the need for effective legal regulation of these activities, both in terms of professional recognition and training. In Spain, recent legislation represents a significant breakthrough in the regulation of yoga instruction, while in Catalonia legislation has been passed on licencing processes and the training of yoga teachers. This article focuses on these processes, raising some key unanswered questions, points of contact and divergence between governments and this services sector, and substantive differences among stakeholders themselves.

Key words: Yoga; Complementary and Alternative Medicine (CAM); Professional Regulation; New Age. 


\section{INTRODUCCIÓN}

En un contexto de progresiva globalización y entrada en la modernidad avanzada se ha producido un fenómeno conocido en las ciencias sociales cómo "reencantamiento del mundo" (Maffesoli 2009), que afecta a un amplio campo de significados difusos y de fronteras inciertas con manifestaciones de muy diversa índole (movimientos fundamentalistas, religiones civiles, culto al patrimonio cultural y a la tradición, religiosidades profanas (música, fútbol, culto al cuerpo...), fiestas populares, el mundo religioso de matriz oriental (Hare-krisna, etc.), la llamada New Age y sus derivados o las formas de conocimiento no científico, herméticas u ocultas). El desarrollo de nuevas espiritualidades (Houtman y Aupers 2007), va acompañado de emergentes movimientos físico-psico-espirituales (Albert y Hernández 2014) que han tenido un claro impacto en ámbitos distintos, pero especialmente en el de la salud y el bienestar debido a su intensa proliferación. Cada día es más frecuente encontrar "alguna enfermera o enfermero que ofrecenreiki a sus pacientes, o algún doctor o doctora que recomiendan meditación, o algún devoto o devota cristianos que han sustituido la oración por el yoga" (Cornejo y Blázquez 2013:12).

El uso de las llamadas Medicinas Alternativas y Complementarias (MAC) ocupa un lugar cada vez más destacado en nuestra sociedad. Se trata de una realidad ya bien visible que se observa materializada en el funcionamiento y en algunos casos, con una larga trayectoria, de los conocidos centros, escuelas, consultas particulares, etc. de terapias alternativas. La proliferación de todos estos espacios terapéuticos camina de la mano de la necesidad de una regulación institucional tanto para el reconocimiento de profesionales que operan en este campo como para su formación (Albert 2014).

Nuestro país, hoy es protagonista de un importante avance en materia jurídica en laregulación de la Instrucción en Yoga a raíz de los Reales Decretos publicados en los últimos años, así como de los procesos de acreditación y de habilitación de los profesores de yoga en el caso catalán. Sin perder de vista que este proceso de regulación no solamente está teniendo lugar en nuestro país; prueba de ello es la noticia que aparecía el pasado 20 de noviembre de 2014, sobre el primer ministro indio, Narendra Modi, por el relanzamiento de una secretaría de Estado dedicada al yoga y a la promoción de la medicina tradicional. La noticia, insiste en que "detrás de esta medida no solo hay espiritualidad, ya que la formación de esta institución coincide con el anuncio del gobierno de establecer una regulación sobre los medicamentos tradicionales. El país quiere expandir su presencia en el mercado global de medicina alternativa, que tiene un valor aproximado de 80.000 millones de euros, según la agencia Reuters" (El País, 20 de Noviembre de 2014).

En este artículo nos planteamos un objetivo descriptivo para el cual vamos a situarnos en los primeros pasos en la regulación de la profesionalización del yoga en la realidad española: cuáles han sido y cómo se han realizado. Para ello, en primer lugar, se presentará una introducción a los orígenes de esta práctica milenaria, deteniéndonos brevemente en su penetración en Occidente, así como en el camino de ida y vuelta entre Oriente y Occidente, lo que nos permitirá contextualizar la práctica del yoga en nuestro país en el momento actual. En segundo lugar, enunciaremos las principales modalidades del yoga desarrolladas y, especialmente la que se ha po- 
pularizado más en Occidente, el Hatha yoga. En tercer lugar, veremos en que consiste la práctica del yoga atendiendo a su proceso de implantación. Y, finalmente, mostraremos como se está produciendo la mencionada regularización del yoga en el caso español. Metodológicamente, se han combinado fuentes primarias y secundarias. Respecto a las primeras, se han realizado entrevistas a profesores/as de yoga, así como a responsables de centros/escuelas de yoga de tres ciudades españolas (Madrid, Barcelona y Valencia) y observaciones en espacios de encuentro (Congresos, festivales, etc.) en la ciudad de Valencia, donde además, se ha tenido la oportunidad de intercambiar impresiones con el sector. Respecto a las segundas, se ha obtenido información a través de un cuidadoso repaso a la normativa jurídica en relación al yoga, así como de noticias de prensa, revistas especializadas (Ahora Yoga, Yoga en red, Tú Mismo, YoguiOla Magazine), y de páginas web/blogs de las principales asociaciones y federaciones del yoga en España.

\section{EL YOGA, UNA PRÁCTICA MILENARIA}

La filosofía India puede dividirse en seis sistemas ortodoxos (nyaya, visesika, sankhya, yoga, mimamsa y vedanta) y tres heterodoxos (carvaka, jainismo y budismo $)^{1}$. La palabra yoga, etimológicamente, deriva de la raíz yuj, "ligar", "mantener oprimido", "uncir", "poner bajo yugo". "Originariamente, estas técnicas, provienen de lejanas prácticas chamánicas neolíticas, oralmente transmitidas y reservadas siempre a una minoría" (AEPY). Desde los albores de la humanidad, los pueblos primitivos han contado con la presencia de sus brujos/as, chamanes/as, etc. personas que poseían la técnica para producir "el trance" o los estados alterados de conciencia, "siendo precisamente el aspecto ascético con el que se entronca el yoga más antiguo, como su desarrollo, prolongación y transformación natural. Fundamentalmente se trata del dominio que el asceta ejerce sobre su cuerpo, sobre sus sentidos, sobre sus deseos y sus actividades mentales, dominándolos, sujetándolos, en él más amplio sentido de disciplina (AEPY).

La práctica milenaria del yoga, en la India, fue integrada por todos los movimientos religiosos, tanto hinduistas como "heréticos" y terminó por absorber y aglutinar toda clase de técnicas espirituales y místicas, desde las más elementales hasta las más complejas. Mahadevan (1998) apunta que no sólo las tradiciones ortodoxas, sino también las llamadas heterodoxas, estaban familiarizadas con las técnicas del yoga. De acuerdo con Eliade (1977), la penetración gradual de la práctica yoga, considerada como un excelente camino de salvación, llevó finalmente a la conquista casi total del espiritualismo indio por el yoga durante un periodo comprendido entre el IV a. c y el IV d. c. Ello coincidió con el empuje de la devoción mística popular. "Efectivamente, la instalación de la técnica del yoga en el mismo corazón del hinduismo² tuvo lugar

\footnotetext{
1 "Aplicado a las escuelas de la filosofía India, el término "ortodoxo" significa "aceptación de la autoridad del Veda" y "heterodoxo" indica "rechazo de esta autoridad" (Mahadevan 1998:16).

2 "Las dos tradiciones espirituales que, tras múltiples tensiones, y al final de una larga tarea de síntesis, terminaron por constituir el hinduismo fueron la tradición religiosa de los indo-europeos de idioma ario y la tradición de los aborígenes (bastante compleja que incluye elementos
} 
en un momento de crisis de la ortodoxia; es decir, en el momento preciso en que la práctica del yoga, validó los movimientos místicos "sectarios"” (1977:145). Parece pues, que "una de las causas principales de esta transformación fue precisamente la necesidad, experimentada por las masas populares, de una experiencia religiosa más concreta, de una devoción mística fácilmente accesible, íntima, personal que las prácticas tradicionales yoguis (populares, barrocas, no sistemáticas) ofrecían justamente una experiencia mística de este género: desdeñando los rituales y la ciencia teológica" (Eliade 1977:146). De forma que el yoga dio respuesta a las necesidades más hondas del alma india, "Señalando una reacción en contra de las teorizaciones metafísicas y los excesos de un ritualismo fosilizado; representaba la misma tendencia hacia lo concreto, hacia la experiencia personal, presente en la devoción popular" (Eliade 1977:341).

Aunque la presencia del yoga puede rastrearse en la tradición oral india, los documentos más antiguos son los Vedas. Se cree que estas escrituras básicas fueron algo escuchado por los bardos de antaño que transmitieron a la posterioridad las ideas que recibieron. "Las verdades védicas fueron descubiertas, no creadas, fueron reveladas a los poetas, no hechas por ellos. (Mahadevan 1998:25). Existe sin embargo, un yoga "clásico", un "sistema de filosofía" expuesto por Pantañjaly en su célebre tratado Yoga-Sutra. Esta obra es el resultado de un esfuerzo enorme, no sólo para reunir y clasificar una serie de prácticas ascéticas y recetas contemplativas conocidas en la India desde tiempos inmemoriales, sino también de ponerlas en valor desde un punto de vista teórico, fundiéndolas, justificándolas e incluyéndolas, en una filosofía, la Samkhya, exaltando el valor práctico de la meditación. De esta forma, gracias a Pantañjaly, el yoga, que era de tradición mística, se convirtió en un "sistema de filosofía". Paralela y posteriormente se desarrollaron otros yogas (desde el brahmanismo, el hinduismo, el budismo, la alquimia, el chamanismo, etc.). Así, tradicionalmente, el término yoga se utilizó para designar todas las técnicas de ascesis y de cualquier método de meditación; aunque utilizadas de forma diferente por las múltiples formas de pensamiento y movimientos místicos indios a lo largo de su historia. El yoga constituye pues, una dimensión específica del espíritu indio hasta tal punto que, en cualquier lugar donde hayan llegado la religión y/o la cultura india, encontramos una forma de yoga.

\section{EL YOGA EN SU CAMINO DE IDA Y VUELTA ENTRE ORIENTE Y OCCIDENTE}

Una de las consecuencias de la colonización de la India por parte del imperio británico fue la relación dialógica de las formas de yoga que se formularon y se transmitieron entre la India y Occidente a partir del siglo XIX en un contexto de flujo de ideas, creencias, y prácticas. De acuerdo con Singleton (2010), las formas cuasi religiosas de

$\overline{\text { drávidicos, munda }}$, protomunda y harappianos). Los indoeuropeos aportaban una sociedad de estructura patriarcal, una economía pastoral y el culto de los dioses del Cielo y de la atmósfera, en una palabra, "la religión del Padre". Los aborígenes pre-arios conocían ya la agricultura y el urbanismo (la civilización del Indo) y, en términos generales participaban de la areligión de la Madre». El hinduismo, tal cual se presenta desde el ocaso de la Edad Media, representa la síntesis de esas dos tradiciones, pero con predomino acentuado de los factores aborígenes: el aporte de los indoeuropeos término siendo asiatizado radicalmenten. (Eliade 1977:342). 
la cultura física europea se infiltraron en la India durante este siglo y dieron lugar a la reinvención de la postura (asana) como la expresión intemporal del ejercicio hindú, desarrollando la dimensión física y postural del yoga. Posteriormente, encontraron su camino de vuelta hacia Occidente donde se identificaron y fusionaron con las formas de "gimnasia esotéricas" que se habían popularizado en Europa y América desde mediados del siglo XIX. Así pues, el yoga que conocemos básicamente hoy en día en Occidente, es el resultado de un intercambio dialógico entre las técnicas de cultivo del cuerpo modernas desarrolladas en Occidente y los diversos discursos de yoga hindú "moderno" que surgieron a partir de las enseñanzas de Vivekananda ${ }^{3}$, pionero en viajar a Occidente e introducir de forma simultánea el yoga y el vedanta en Estados Unidos e Inglaterra a través de sus conferencias, seminarios y discursos privados de doctrina vedanta. Cabe mencionar también, en está difusión del yoga por parte de maestros indios a Paramahansa Yogananda, prolífico escritor y conferenciante, creador de un renombrado y vasto volumen de obras sobre el yoga, el arte de llevar una vida equilibrada y la unidad que constituye el fundamento de todas las religiones. Su obra más difundida, Autobiografía de un Yogui (1945), tiene el valor de ser uno de los pocos libros escritos en inglés no por un extranjero y que hoy en día sigue siendo reconocido en todo el mundo como una obra clásica de literatura espiritual ${ }^{4}$.

El yoga, en su difusión en el mundo occidental, ha sufrido una transformación radical en respuesta a las diferentes visiones del mundo, lógicas, predisposiciones y aspiraciones de las audiencias modernas. Estas formas modernas han sido el resultado de un replanteamiento de las prácticas y marcos de creencias en la propia India en los últimos 150 años, en respuesta a los encuentros con la modernidad y Occidente. Así pues, en la línea de Singleton (2010), todos los yogas, dentro y fuera de la India, llevan las huellas de este intercambio dialéctico. E incluso podemos señalar, como matiza éste autor, que la práctica postural moderna del yoga no es sinónima de los anteriores yogas desarrollados en la India (el yoga clásico de Patañjaly; el yoga de la Bhagavad Gita, etc.) pues cada uno de ellos tiene su propio significado y su propio origen. Así nos lo cuenta uno de nuestros entrevistados:

Nosotros más bien era un espíritu basado en las enseñanzas de Patanjaly pero el yoga enriquecido con las aportaciones tanto de la ciencia de Occidente, la terapia corporal de occidente que tiene mucho que aportar en cuanto a pedagogía de enseñanza, en cuanto a forma de trabajar el cuerpo de forma diferente, en cuanto a estudio a la anatomía y la fisiología sobre el yoga. Métodos pedagógicos como los reguladores para las posturas, compensaciones, atención individual en la que nosotros nos hemos convertido en especialistas (Sadhana)

La práctica postural moderna puede por tanto, considerarse en sus propios términos y no en comparación negativa a otras tradiciones yoguicas (Singleton 2015:15). Así lo expresa una profesora de yoga:

\footnotetext{
${ }^{3}$ Vivekananda (1863-1902) fue un pensador, místico y líder religioso indio, discípulo del místico Ramakrishna.

${ }^{4}$ Fundó en 1920 la Self-Realization Fellowship con la finalidad de difundir mundialmente sus enseñanzas. Frawley (1996) afirma "se puede decir que Yogananda es el padre del yoga en Occidente: no del yoga meramente físico que se ha hecho tan popular, sino del yoga espiritual, es decir, de la ciencia de la percepción del Ser, que es el verdadero significado del yoga" (Citado en Yogananda 2008:XXVII)
} 
Yo creo que actualmente los mejores profesores de yoga se encuentran aquí en Occidente, se trabaja con mucho rigor. Se ha perdido la esencia, la alegría y la manera de hacer que tienen ellos (orientales) también. Aquí se trabaja de una manera muy occidental, muy mental, pero hay muy buenos profesores de yoga. (Profesora de Yoga)

En la segunda mitad del siglo XX, Occidente fue testigo de un importante crecimiento en el interés por el yoga que se materializó en una gran proliferación de manuales prácticos, los cuales ayudaron a alinear el yoga con las nociones occidentales de deporte y ejercicio. Durante la década de los 60, toda una generación de jóvenes americanos y europeos se interesaron por el yoga, ya que éste se constituyó en un icono contracultural (baste recordar el romance espiritual de los Beatles con el yogui Maharishi Mahesh), reforzando la posición de yoga en la psique popular e inspirando a muchos de estos jóvenes a unirse a la "ruta hippy" hacia la India en busca de filosofías y estilos de vida alternativos.

En las décadas siguientes, los 70 y los 80, el establecimiento y la expansión de un número significativo de escuelas e institutos dedicados a la práctica del yoga comportaron una clara consolidación del yoga en Occidente. Fue, durante este período cuando se produjo un claro acercamiento del yoga con la floreciente New Age. Para mediados de los años 90, el boom del yoga se había convertido en una empresa comercial importante, con altos niveles de comercialización y mercantilización que desarrolla diferentes sistemas posturales (Singleton 2010:20). Se produce pues, un proceso constante de expansión e implantación de la práctica del yoga.

\section{MODALIDADES EN LA PRÁCTICA DEL YOGA}

La primera impresión que podemos llevarnos, cuando nos acercamos al mundo del yoga es la de que no existe un yoga, sino muchos. Con sus grupos, técnicas, interpretaciones, escuelas, etc... Tal y como aparece en la página web de la Asociación Española de Practicantes de Yoga (AEPY), existe un yoga integral, pero en él, hallamos diferentes modalidades; se refieren así al yoga devocional (Bhakti yoga) ; al yoga de la acción desinteresada (Karma yoga) ${ }^{6}$; al yoga del conocimiento (Jñana yoga); al yoga del poder latente en todo ser humano (Tantra yoga $)^{8}$; al yoga del sonido (Nada

\footnotetext{
5 Enseña a transformar las emociones en devoción. Actúa en el amor a la divinidad. Es el sendero del amor a Dios y la devoción. La unión con lo divino y con el gurú o maestro espiritual. Es el camino de la mística en donde todo proviene de la gracia de Dios. Es un yoga muy ritual y contemplativo.

${ }^{6}$ Propone realizar los deberes y los actos sin apego a los frutos de la acción. Servir a todos desinteresadamente. El deber cumplido sin interés, sin egoísmo, sin apego. Es una de las grandes enseñanzas del Bhagavad Guita, el evangelio clásico de la acción desinteresada. Este yoga va disolviendo el ego. El Karma-yogui, actúa por solidaridad pero sin protagonismo. Es el amor permanente a todos y todo sin esperar nada a cambio. Según la ley del Karma, todo acto tiene su reflejo, su consecuencia. Actúa sobre la emotividad y controla la actividad.

7 Es el yoga de la meditación, de la sabiduría a través de la auto observación y la conciencia. El estudio y la reflexión. De la obediencia a las instrucciones del maestro para obtener el control mental y el discernimiento que nos conducirá a tomar conciencia ecuánime de la realidad con una inteligencia despierta y llena de creatividad.

${ }^{8}$ Entiende al ser humano y al cosmos como un todo unido, una inmensa red unificada. Este yoga, íntimamente relacionado con la naturaleza y sus energías, propone, mediante técnicas de
} 
yoga o Mantra yoga $)^{9}$. Podemos citar muchos otros, como el yoga de la sencilla y espontánea naturalidad (Sabaja yoga); el yoga del linaje de los Siddhas (Siddha yoga); el yoga de los Bauls de Bengala; el sistema de Vinyasa Ashtanga y su yoga de la energía; el lucrativo sistema de Yoga $B_{1 k r a m}^{10}$, y un largo etc. Además, dada la riqueza y variedad cultural India, la pervivencia de sus tradiciones, la mezcla de escuelas, maestros, religiones, cultos, ideas etc. hace que muchos de estos yogas se entremezclan en métodos personales o en técnicas eclécticas. Es el caso del yoga Iyengar, resultado de toda una vida dedicada a la práctica del yoga por B.K.S. Iyengar, convertido en una personalidad máxima en el mundo del yoga actual, al cual se refiere como un arte, una ciencia y una filosofía.

Sin embargo, el yoga más difundido en Occidente y el más popular es el conocido como Hatha Yoga, desvinculado de las raíces religiosas que le dieron su razón de ser (Díez de Velasco 2002). Es el yoga de la fortaleza y armonía del cuerpo referido a las dos cualidades, solar y lunar del fluido o prana vital del cuerpo. Pretende, no sólo fortalecer el cuerpo, sino también armonizar y equilibrar todo el sistema nervioso, "basado en el control del cuerpo por medio de posturas (asana) y técnicas de control de la respiración (pranayama), actúa como una preparación para el acceso a formas meditativas del yoga" (Díez de Velasco 2002: 229). De esta forma, actúa fundamentalmente sobre el cuerpo físico y la respiración procurando una salud perfecta. Tal como nos recuerda Eliade (1977), la aparición del Hatha Yoga está unida al nombre de un asceta que vivió en el siglo XII, Gorakhanath, fundador de una orden, la de los Kanphata-yogui y que llamaron Hatha Yoga a su propia disciplina, pero esa palabra pronto pasó a designar el conjunto de recetas y disciplinas tradicionales mediante las que se llegaba a dominar perfectamente al cuerpo.

Hatha Yoga, Jñana Yoga y otras de carácter ritual, esotérico e incluso sexual para despertar la primera Shakti o energía en forma de suprema fuerza de luz y calor dentro del cuerpo, que se halla en la base de la columna vertebral, simbólicamente enrollada en forma de serpiente (Kundalini). Este poder serpentino al ser alterado (Kundalini Yoga) se desplaza por la columna vertebral y otras ramificaciones o canales sutiles de energía llamados tradicionalmente nadis atravesando a su vez plexos o centros de energía (chakras) que al ser activados permiten desarrollar potencialidades humanas latentes, alcanzando elevados niveles de conciencia y realización personal. Obtener ese estado en que se produce la disolución, la absorción en el todo se conoce también como el yoga de la disolución (Laya Yoga) o sendero de reintegración mediante la voluntad y el esfuerzo en la concentración. Dentro de lo que es el tantra yoga, hay que citar los yantras o diagramas geométrico-artísticos de carácter mágico-ritual. Símbolos, mandalas, signos astrológicos, esotéricos etc. Ocasionalmente son empleados como el soporte inicial para fijar la atención e ir preparando la entrada en la meditación.

${ }^{9}$ Esta referido, fundamentalmente, al poder del sonido o la vibración, soporte final de la materia. El OM, la sílaba sagrada, expresión simbólica del sonido primigenio, de la vibración original, a partir de la cual se ha manifestado todo lo existente. Y por extensión la palabra. Tradicionalmente a la palabra se le otorga un poder no solo esotérico sino real, existiendo técnicas muy depuradas de yoga, especialmente en India, basadas en la repetición concentrada (Japa Yoga) de un sonido con especial significación (mantra), normalmente destinado a provocar un estado contemplativo o transmisión de poder.

${ }^{10}$ Noticia en el periódico El País, 11 de junio de 2014 «¿Qué tienen en común Lady Gaga, Elena Salgado y Barack Obama?». El Bikram yoga se ha convertido en el exigente método de los famosos para ponerse en forma que se práctica añadiendo calor $\left(40^{\circ}\right)$ lo que permite calentar los músculos y eliminar un gran número de toxinas a través de la piel. 
En esta práctica postural moderna del yoga, el cuerpo es considerado como el instrumento más seguro y completo de que disponemos. Debe, por tanto, ser conservado el mayor tiempo posible en un estado perfecto ${ }^{11}$. Pero esta dominación del cuerpo no es de orden higiénico ni atlético, tampoco puede ser confundido con la gimnasia. El maestro B.KS. Iyengar señala que algunos olvidan el aspecto psicológico de la definición del Hatha Yoga y solo toman en cuenta el aspecto fisiológico cuando dicen que significa "yoga físico". Tha (luna), en realidad significa la conciencia, que, como ella, tiene diferentes ciclos o fluctuaciones. En cambio, $\mathrm{Ha}$ (sol) brilla constantemente, sin decaer jamás. Hatha Yoga, por tanto, es la ciencia que proclama que la mente fluctuante debe ser elevada al nivel del sol que nunca decae. Según este maestro, "el yoga consiste en desarrollar el cuerpo para que alcance el nivel de la mente, y así cuerpo y mente se conviertan en uno. Cuerpo y mente se coordinan para experimentar la "fuente de la vida". Considero que cuando se trabaja el cuerpo exterior, estructural, físico, los huesos, las articulaciones y los músculos, se llega a comprender el cuerpo y la mente interiores. A través de ello he entendido el elixir de la vida" (Battles 2012:159-160). Para este maestro, "no hay absolutamente ninguna diferencia entre los distintos tipos de yoga, aunque los autores le hayan dado diferentes nombres a un mismo contenido; es como si quisiéramos dar diferentes nombres a los pétalos de una flor" (Battles 2012:170).

\section{IMPLANTACIÓN DEL YOGA EN ESPAÑA}

Como ya se ha señalado, la penetración del yoga en Occidente es anterior, pero para rastrear su implantación y su extensión en el caso español, tenemos que situarnos en los años 70 del pasado siglo en contexto de profundos cambios políticos y sociales tras cuarenta años de dictadura. Durante esos años de lucha por la democracia y de aperturismo propios de la transición española, llegaron a nuestro país destellos de los movimientos contraculturales que compartidos y ampliados por la movida hippy produjeron luchas intergeneracionales provocadas por el hastío que los jóvenes sentían hacia una sociedad cada vez más tecnocrática y sofisticada. En palabras de Roszak (1978), provocaron la búsqueda de alternativas con un interés progresivo por los misticismos orientales, las drogas psicodélicas y por las experiencias de vida comunales.

Así, fueron muchos los jóvenes que viajaron a la India en busca de filosofías y estilos de vida alternativos y entre otras cosas, se encontraron con el yoga, una práctica que trata de desarrollar las facultades del ser humano para permitirle afrontar su realidad exterior a través de la experiencia del sí mismo. Como condición previa, el yoga es una ruptura de los lazos que unen el espíritu al mundo. Si etimológicamente yuj quiere decir "ligar", es sin embargo evidente que el "lazo" al que esta acción de atar debe llevar.

\footnotetext{
${ }^{11}$ En esta enfática apreciación del cuerpo humano y de sus posibilidades, podemos distinguir dos orientaciones diferentes y convergentes a la vez: 1) la importancia otorgada a la experiencia total de la vida considerada como parte integrante del sadhana (esta es principalmente la posición de todas las escuelas tántricas) y 2) la voluntad de dominar al cuerpo para transformarlo en un cuerpo divino (esta es principalmente la posición del Hatha Yoga) (Eliade 1977:223).
} 
Además de su estructura de iniciación, el yoga se caracteriza por su aspecto práctico. El yoga no se aprende solo, es necesaria la dirección de un maestro (gurú). El yoga se plantea como una serie de experiencias metodizadas y ascendentes, que requieren la guía de alguien que haya recorrido ese camino para orientar a un individuo, porque de otro modo caemos en simples abstracciones, caemos en un nihilismo que no tendría ningún sentido (Requena 1998). Así, «el yogui debe practicar una serie de "disciplinas" (niyama) corporales y psíquicas: la pulcritud, la serenidad (samtosa); la ascesis (tapas); el estudio de la metafísica yoga y el esfuerzo para hacer de Dios (Ivasa) el motivo de las propias acciones" (Eliade: 1977:60).

Algunos de estos jóvenes que tuvieron estas experiencias, a su vuelta fueron los que iniciaron la práctica del yoga en nuestro país. En este sentido, cabe destacar, en primer lugar como referente indispensable a Ramiro A. Calle, quien no solo viajó a la India en sus años de juventud, sino que lo ha continuado haciendo a lo largo de toda su vida ${ }^{12}$. Convertido en maestro y escritor de yoga, ha estudiado en profundidad los efectos terapéuticos de las psicologías orientales y de los aportes de la meditación al psicoanálisis, la psicoterapia y la neurociencia. Fue el primero en promover investigaciones médicas sobre el yoga como terapia en España, en colaboración con destacados médicos y especialistas. Sus libros de orientalismo y de autoayuda han sido leídos por millones de personas, principalmente de habla hispana. También ha realizado una importante labor de divulgación de las técnicas del yoga, participando en numerosos programas de radio y televisión.

Nos interesa, sin embargo, destacar su carácter pionero en la enseñanza del yoga en España, disciplina que imparte desde hace más de 40 años en el centro de yoga y orientalismo "Shadak" que dirige desde 1971 en Madrid. Ha enseñado de forma directa yoga, relajación, y técnicas de meditación a más de quinientas mil personas, ayudando a las mismas a prevenir y combatir problemas psicosomáticos. En este centro, la formación de profesores de yoga se realiza a aquellas personas que llevan años aprendiendo.

Otro referente importante, también por su carácter pionero a destacar es Carlos Fiel, quien en sus viajes a la India aprendió y profundizó en distintos aspectos del yoga y otras enseñanzas que van desde la meditación Vipassana, la tradición budista tibetana, pasando por su ordenación como monje zen, etc. Es fundador de Sadhana, que comienza su andadura en el año 1973 y que se conforma como Asociación de yoga Sadhana dos años más tarde siendo su presidente y director pedagógico. Posteriormente, en el año 1980 creará y estructurará la Escuela de formación de profesores de yoga, reconocida por la Unión Europea de Yoga, de la que es presidente y director pedagógico, formador de profesores y de formadores de yoga. En la actualidad Sadhana está implantada en algunas de las principales ciudades de nuestro país (Madrid, Barcelona, Sevilla, Valencia y San Sebastián) así como en Italia, Francia y Suiza, con más de 600 enseñantes.

En marzo de 1987 nace Sadhana en Valencia, centro pionero en traer a Valencia disciplinas y profesionales relacionados con una visión holística del ser humano, destacando en la enseñanza del yoga y centro de referencia. Dirigida actualmente por Antonio Triguero, miembro fundador del centro en los años 80, señala:

${ }^{12}$ Para conmemorar el centenar de ocasiones que ha estado en Oriente ha publicado el libro titulado "100 viajes al corazón de la India". 
...hay de nuevo, hay un resurgir, nosotros fíjate, que llevamos muchos años haciendo yoga. Ahora hay un resurgir muy interesante, que es el de las generaciones jóvenes, de las madres que tienen hijos que quieren: primero, acceder a un embarazo consciente, a un parto consciente y luego también tener una crianza más consciente. Para eso el yoga se está desarrollando mucho. Ahora el yoga para niños hay una auténtica eclosión, incluso centros que se están abriendo y el yoga en familia. Una verdadera eclosión de eso... [...] ...Yo creo que la necesidad, quizá encontrar formas de estar con la familia de más calidad, con los niños. La sociedad en general es algo muy materialista, si ya lo era, ahora más. Entonces buscar cosas que unen, el yoga es unión. Si tu estás con tus hijos haciendo esas cosas, muchas veces las madres no tienen ese contacto, si trabajan, los niños se quedan en la guardería, los recoges muy tarde, o te los recoge la abuela y hay poco contacto y cuando llegas a casa es trabajo y más trabajo... yo creo que hay una necesidad de tener y de retomar un contacto, que a lo mejor antes se tenía pasando horas juntos, en familia... ahora pienso que se necesita un desarrollo de otra manera y el yoga es una manera... (Sadhana)

Pero será, fundamentalmente, durante la década de los noventa del siglo pasado y la siguiente, cuando se produce una verdadera eclosión y muchos centros especializados en yoga abrieron sus puertas, fruto de la formación y de las enseñanzas recibidas de los centros pioneros como el descrito anteriormente.

...yo desde los 19 años que tome contacto con el yoga no he dejado de aprender, estuve en varias escuelas, principalmente aquí en Valencia, en Sintagama, en Yoga Yoga... pero también en Madrid y en Barcelona haciendo formación y aprendiendo. Hasta el año 2000 aquí en Valencia había muy pocos centros, Gobinde se inauguró en septiembre del 2006... (Gobinde)

Estos centros no solamente invitaban a la práctica del yoga, sino que además, ofrecían la posibilidad de una formación más profunda para convertirse en profesor de yoga. Se produce así, una proliferación de profesorado en este campo que contribuye a su expansión e implantación. Juan Almirall, presidente de la Federació Catalana de Ioga asegura, además, que el auge de maestros de yoga hace que los cursos de formación de profesores sean los que están financiando a muchos de los centros. Así, muchos de estos noveles profesores de yoga accedieron a espacios deportivos (polideportivos y gimnasios) principalmente, pero también educativos escuelas de adultos) y culturales (casas/casales de juventud y casas de cultura) popularizando el yoga como una práctica saludable especialmente antiestresante y relajante, cada vez más recomendada por expertos de la salud. "El yoga ha crecido. Ha dejado de ser algo místico. Ahora, hasta lo recomiendan los médicos. Es algo normal que tiene muchísimos adeptos" (El País, 29 de septiembre de 2013).

Hoy, la práctica del yoga se ha escapado de los pequeños centros exclusivos para abrirse al gran público. Organizan festivales, congresos, encuentros y quedadas multitudinarias ${ }^{13}$ dando visibilidad y popularizando la práctica del yoga.

... Bueno, desde el centro organizamos actividades, ofrecemos clases gratuitas, por ejemplo, todos los días a las 6h.30' de la mañana se hace una meditación, abierta para quien

\footnotetext{
13 En la ciudad de Valencia se celebran de forma permanente, encuentros, "quedadas", conciertos tanto al aire libre (el rio, la playa) como en establecimientos cerrados para practicar el yoga. También, se ha consolidado la celebración anual del importante Congreso Mediterráneo de Yoga que ya va por su tercera edición y el Spring yoga Festival que durante este año 2015 realizó su segunda edición.
} 
quiera venir. Con la gente que va pasando por el centro, o gente que toma contacto con el yoga, pues se van generanado cosas, y nos gusta hacer cosas que respondan a estos intereses... el año pasado organizamos el I Spring Yoga Festival... vino mucha gente durante todo el fin de semana. Ahora estamos preparando el de este año, también lo haremos en Mayo... organizamos también retiros, algunos más específicos de las formaciones y otros de carácter más abierto, el año pasado hicimos cuatro... también el año pasado hacíamos una quedada cada mes, y seguimos haciéndolas, sin publicidad ni nada, lo lanzamos, invitamos a la gente que conocemos y se va difundiendo, son gratuitas y vamos variando los lugares, siempre aquí en Valencia, plazas, parques... el mes pasado hicimos una Bhakti (yoga devocional), ya habíamos organizado otra anteriormente, también abierta y gratuita, 12 h. seguidas de meditación, yoga, mantras, con músicos en directo... (Gobinde)

En esta línea, resulta ilustrativa la noticia "La práctica del yoga se expande", publicada el 29 de septiembre de 2013, en el periódico El País, a raíz del acto en el que cerca de 2000 yoguis se reunían en el Arc de Triomf en Barcelona para practicar esta disciplina. "A falta de datos oficiales, los profesionales del sector certifican que el número de practicantes y centros de yoga no ha hecho más que aumentar de forma desorbitada en los últimos cinco años. Almirall, presidente de la Federación Catalana de Yoga, calcula que puede haber más de 300 centros solo en Barcelona”. Además, continua la noticia, empieza a imponerse con fuerza el turismo de yoga, con clientes extranjeros que demandan paquetes vacacionales que incluyan sesiones de yoga durante su estancia en la ciudad ${ }^{14}$. Expansión que también se da en otros países y que tiene su referente europeo en el Festival de Yoga que se celebra anualmente en la localidad francesa de Soulogne desde el año 1977 . «El año 2009 el público asistente aumento un 25\% respecto de años anteriores y en 2010 este porcentaje se duplicó" (Prat 2012:137).

\section{REGULACIÓN Y NORMATIVA EN ESPAÑA}

Parece obvio, que tras la implantación y popularización del yoga en nuestro país se abra una vía de regulación de dicha práctica. Así lo mantiene Juan Almirall, presidente de la Federació Catalana de Ioga.

...Yo lo que pienso es que más tarde o más temprano este proceso se hubiera puesto en marcha, ¿por qué? Sencillamente por el éxito que está teniendo el yoga. Lo que no puede ser es una cosa de éxito que practican millones de personas pues que se haga de forma no regularizada... una cosa que se ha popularizado tanto. Bueno, nos ha cogido como nos ha cogido... (FCI)

Nos interesa, sin embargo, destacar como se ha realizado este proceso. Así, en el camino de la regulación de la práctica del yoga en España tienen un papel protagonista Sadhana, la Asociación Española de Practicantes de Yoga (AEPY) la Asociación Española de Kundalini Yoga (AEKY), todas con sede en Barcelona. Se trata de asociaciones que cuentan con una larga trayectoria en la formación de profesores de yoga

\footnotetext{
${ }^{14}$ En la noticia especifica que la agencia de viajes Xuan-LanTrinh oferta talleres para turistas: "Es gente que no quiere dejar de practicar ni en vacaciones y además, aprovechan que están de descanso, relajados, para hacer yoga a primera hora".

${ }^{15} \mathrm{El}$ año 2011 se realizó en un nuevo emplazamiento debido a la gran afluencia de público.
} 
y que son un claro referente en nuestro país en esta práctica. Desde estas asociaciones se vio la necesidad de regular dicha formación

\begin{abstract}
...Nosotros somos una federación que se llama Sadhana, luego hay una asociación que se llama AEPY que es la más antigua que hay en España y luego hay toda una constelación de asociaciones y federaciones y uno se puede formar pues en la asociación que quiera. Entonces claro, no hay regulación, cualquiera puede decir que es profesor de yoga, con formación, sin formación, con cualificación, sin cualificación y decir lo que le dé la gana. Entonces, se ve la necesidad de regular eso... (Sadhana)
\end{abstract}

Otros profesionales del yoga, interpretan más bien, que de lo que se trataba era de poner límites, de acotar la posibilidad de obtener una formación como profesor de yoga. Puesto que la constelación de espacios que ofrece dicha formación y que depende prácticamente de ella para sostener el centro no ha hecho más que aumentar. "Almirall asegura que el auge de maestros de yoga hace que los cursos de formación de profesores sean los que están financiando a muchos de los centros: en Barcelona es impresionante, están saliendo profesores de debajo de las piedras" (El País, 29 de Septiembre de 2013). En esta línea se pronuncian voces más críticas al respecto:

estas asociaciones también tenían intención de limitar o de intentar limitar un poco la formación de profesores de otras escuelas que también vieron el negocio en la formación. (Profesor de yoga)

Las asociaciones citadas mantuvieron reuniones y realizaron gestiones de contacto en un primer momento con la Generalitat de Cataluña, a la que presentaron formalmente la petición de regulación de la formación del yoga para futuros profesores. Sin embargo, la Generalitat Catalana no tiene competencia para legislar en materia de programas formativos, pues esta pertenece al Ministerio de Educación. Así, la petición fue transferida, en concreto al Instituto Nacional de las Cualificaciones (INCUAL) adscrito al Ministerio de Educación que tiene, entre otras competencias, el desarrollo, la elaboración y el mantenimiento actualizado del Catálogo Nacional de Cualificaciones Profesionales y la elaboración de los instrumentos de apoyo necesarios para la evaluación y acreditación de las competencias profesionales adquiridas a través de la experiencia laboral y vías no formales de formación. Así, la cualificación de Instrucción en Yoga fue elaborada por el INCUAL con la colaboración de expertos del sector; las Comunidades Autónomas participaron en su elaboración a través del Consejo General de Formación Profesional en las fases de solicitud de expertos para la configuración del Grupo de Trabajo de Cualificaciones, contraste externo y en la emisión del informe positivo realizado por el Consejo General de Formación Profesional, necesario y previo a su tramitación. Finalmente, verá la luz como Real Decreto en el Boletín Oficial del Estado, el 4 de Agosto de 2011, 1034/2011, por el que se complementa el Catálogo Nacional de Cualificaciones Profesionales, mediante el establecimiento de cinco cualificaciones profesionales de la familia profesional Actividades Físicas y Deportivas ${ }^{16}$, así como sus correspondientes módulos formativos de Forma-

\footnotetext{
${ }^{16}$ Conjunto de cualificaciones en las que se estructura el Catálogo Nacional de Cualificaciones Profesionales, atendiendo a criterios de afinidad de la competencia profesional. La relación de familias profesionales se establece en el Real Decreto 1128/2003, de 5 de septiembre, por el que se regula el Catálogo Nacional de Cualificaciones Profesionales y en el Real Decreto 1416/2005,
} 
ción Profesional; entre las que se encuentra la de Instrucción en Yoga. No obstante, son muchos los profesionales consultados que manifiestan su total desconocimiento durante los inicios de este proceso.

... pero si nadie sabía nada, cuando nos hemos enterado la ley ya estaba hecha... (Profesora de Yoga)

...Ahora por ejemplo se abrió el espacio para que se presentaran los formadores que iban a ser los oficiales pero solo se enteraron en Cataluña. Entonces solo se presentaron formadores de Barcelona... (Profesora de yoga)

De esta forma, se reconoce la práctica del yoga como una actividad deportiva, cuestión ésta ampliamente criticada por el sector, pues entienden que el yoga no es un deporte y de forma únanime cualquier persona dedicada al yoga manifiesta que "el yoga no es gimnasia, no es competitivo, no tiene que ver pues con el deporte porque no hay competición". Así, la Federació Catalana de Ioga, en su página web expone que es precisamente la diversidad presente en el sector y su dificultad en articular una sola voz lo que ha provocado que el yoga finalmente se regule como una práctica deportiva:

Somos conscientes que este es un sector muy desunido, primero porque nadie se gana muy bien la vida con el yoga y que por tanto hace falta trabajar mucho y queda poco tiempo para otras cosas; segundo, porque yogas hay muchos y eso marca diferencias que nos dificultan navegar en el mismo barco. Por eso, porque el sector no ha conseguido unirse y hablar a través de una única voz, la administración ha regulado el yoga como su fuera un deporte. (FCI)

Insisten además, que es precisamente la falta de conocimiento en esta materia por parte de la administración la que ha provocado la calificación del yoga como un deporte.

... Solamente una profunda ignorancia ha permitido que la Administración califique el Yoga como una actividad deportiva... (FCI)

Y, expresan su total desacuerdo en cómo se está realizando el proceso de regulación:

Estamos total y absolutamente en contra no solamente del proceso Acredita't, sino también de la regulación de la formación en instrucción en yoga del Ministerio de Trabajo y de la consideración del yoga como un deporte, pues nos parece que eso es un freno al gran desarrollo espiritual y social que puede aportar el yoga a nuestra sociedad y cualquier intento de regularlo es una limitación al proceso de evolución espiritual. (FCI)

Son numerosas las voces que de una u otra forma manifiestan que el ideal del yoga consiste en vivir en un presente eterno, fuera del tiempo. Así, el practicante de yoga se va unificando, se va uniendo a sí mismo, va juntando sus facultades sen-

$\overline{\text { de } 25 \text { de noviembre }}$, que lo modifica. Por área profesional se entiende un ámbito de actividades productivas afines que conlleva un desarrollo profesional homogéneo dentro de una familia profesional y de los conocimientos y habilidades que son necesarios para alcanzarlos. La relación de las áreas profesionales son las que figuran en la Resolución de 12 de marzo de 2010, del Servicio Público de Empleo Estatal, por la que se establece el procedimiento para la inclusión de nuevas especialidades en el fichero de especialidades formativas. 
soriales para hacerlas converger con las psíquicas, las mentales y las espirituales, hasta tener una conciencia unificada, un yo como persona que confrontará con un yo total $^{17}$. En el blog de la Federació Catalana del Ioga aparece de la siguiente forma:

... El yoga es un conjunto de prácticas místicas utilizadas desde muy antiguo en la India y otros países de Oriente, no solamente incluye actividades corporales, sino prácticas de estabilización de la respiración y la mente, con el fin de conseguir estados contemplativos y espirituales, como es el Samadbi... [...] "... hay una práctica espiritual del yoga que directamente afecta al derecho fundamental de libertad ideológica, religiosa y de culto. Por otra parte, hay un yoga devocional, otro puramente filosófico, contemplativo y místico, un yoga que persigue finalidades espirituales de realización personal, que no tiene nada que ver con las finalidades deportivas... (FCI)

Tal como se manifiesta en el sector, el yoga, claramente implica una dimensión espiritual, otra filosófica y finalmente, una física.

Porque no se montan entidades religiosas que reivindiquen la parte religiosa del yoga, porque el yoga esta dentro de una tradición espiritual. También hay una parte del yoga que es filosófica y por supuesto la física... los clubes deportivos, las entidades filosóficas y las religiosas todas pueden reclamar una parte del yoga porque son tres ámbitos en los que el yoga está presente. (Profesor de Yoga)

Y es precisamente esta última, la parte física, la conectada con el bienestar y la salud, la que se ha expandido y se ha popularizado.

La gente del yoga está que trina, pero es lógico que se halla hecho de esta forma, el yoga ha dado el tirón que ha dado porque se ha incorporado en gimnasios, porque la gente lo toma como una actividad física. (Profesor de yoga)

Resulta lógico por lo tanto, que se haya regulado como un deporte pues es como la mayoría de la gente entiende el yoga, como una actividad física. Así se expresa por parte de algunos profesionales del sector:

... A la gente lo que le mola del yoga es la cosa aeróbica, entonces la gente se ha cogido al yoga dinámico... (Profesora de yoga)

En abril de 2012, se reúnen en Madrid, una veintena de asociaciones de yoga ${ }^{18}$ del territorio español con la voluntad de aglutinar en una sola voz el yoga español y

\footnotetext{
${ }^{17}$ Es decir, logrará la conciencia de "Atman", para integrarla a la conciencia de "Paramantman" y lograr así el estado de yoghi, y el que está haciendo el trabajo para lograrlo es el practicante de yoga. (Requena 1998).

${ }_{18}$ Asociación Internacional de Profesores de Yoga, Ma Teresa Poch López de Briñas; Asociación Española de Practicantes de Yoga, NelicGironès Manzano; Asociación Centro de Yoga SivanandaVedanta,Gopala (José María Márquez Jurado); Escuela Internacional de Yoga Mayte Criado Regidor; Asociación Centro de Yoga Milarepa-Centro de Estudios Yoguicos, Alejandro Torrealba Álvarez; Asociación de Yoga Sadasiva, Juan Antonio Sánchez Payán; Asociación Profesional de Profesores de Yoga Asmita; Saúl Martínez Calvo; Asociación de Yoga Sanatana Dharma Joan Gibert Canyadell; Asociación Nacional de Profesores de Yoga y Expertos en Indología, Francisco Luis Escuela (Swami Tirthananda); Asociación Española Yoga en la vida cotidiana, María Almudena Basanta Albares (Lila); Asociación Española de Yoga Iyengar, José María Vigar Gutiérrez; Asociación Internacional Yoga Mandir, Gloria Rosales Cantarell; Asociación ETY Viniyoga España, Jaume Grau Font; Asociación Yoga Síntesis, Julián Peragón Casado (Arjuna); Asociación Es-
} 
crean la Federación Española de Entidades Formadoras de Yoga (FEDEFY), con el objetivo de crear una plataforma de diálogo para aglutinar a todas las entidades sin ánimo de lucro bajo cualquier linaje y/o escuelas que se dediquen a la formación profesional de instrucción en yoga, para colaborar en la aplicación de la normativa sobre la cualificación profesional de Instrucción en Yoga. Aunque no todos los profesionales del sector se sienten identificados, ni aceptados en dicha federación

... Ahora mismo es un momento delicado, los requisitos están ahí, nadie sabe quién va a poder cumplir esos requisitos y desde lo que es la federación que se ha creado para poder regular esto, decidir quién entra, quien no entra es un poco extraño, para poder acceder a esa federación tú tienes que tener dos padrinos que te den el visto bueno para entrar y si no, no puedes entrar... está siendo muy confuso todo en cómo se está haciendo. Está muy claro lo del BOE, los requisitos que se piden, pero luego las formas no están quedando claras... (Profesora de yoga)

Pretenden pues, y así lo explicitan en su página web, ser un interlocutor válido y representativo del sector ante las administraciones públicas del Estado y cualquier otra de ámbito internacional, y los ciudadanos ${ }^{19}$. Sin embargo, los profesores de yoga manifiestan:

... se regulo de tal manera que ahora, no pueden estar dentro muchos de ellos, los mismos que estaban por la regulación, porque se les fue de las manos... llego al gobierno y desde el gobierno ya empezaron a hacer sus cosas... ninguno de los centros de yoga cumple con los requisitos de instalaciones, por ejemplo... (Profesora de yoga)

Ese mismo año, se publica el Real Decreto 1076/2012, de 13 de julio, por el que se establecen seis nuevos certificados de profesionalidad, entre ellos el de Instrucción en Yoga, de la familia profesional actividades físicas y deportivas que se incluyen en el Repertorio Nacional de certificados de profesionalidad ${ }^{20}$. Y será a partir de este momento, cuando se produzca un antes y un después para las personas dedicadas al yoga. Si bien el Real Decreto anterior suponía una regularización en la formación no resultaba en si mismo incompatible, puesto que no afectaba a la práctica que realizaban los profesores de yoga ni a las actividades que se realizaban en los centros de yoga. Se había producido una regulación oficial de la formación en yoga, pero lo cierto es que paralelamente, los centros y profesionales del sector podían seguir operando como hasta la fecha independientemente de la regulación oficial, puesto que la aplicación de esta regulación no se había producido, ni se sabían cuales eran

pañola de Kundalini Yoga, Andrea Märtens Alfaro; Associació Yoga Studio Barcelona, Xavier Sola Casas; Asociación de Yoga Naradeva, Luís Luna García; Federación Española de Yoga Satsanga, José Antonio Elosua Oliden; Asociación Nacional de Yoga Sadhana, Carlos Fiel Jareño y la Federación de Yoga Yoguismo, José Luís Hernansáiz Martínez.

${ }^{19}$ Podrán pertenecer a la Federación aquellas asociaciones que no tengan ánimo de lucro y que no estén sometidas a un régimen asociativo específico, previamente inscritas en el correspondiente registro, que tengan interés en el desarrollo de los fines de la Federación y que realicen cursos de formación de Instrucción en Yoga, adaptados al Anexo DCXVI, del Real Decreto 1034/2011, de 15 de julio, por el que se complementa el Catálogo Nacional de Cualificaciones Profesionales.

${ }^{20}$ Estos certificados acreditan el conjunto de competencias profesionales que capacitan para el desarrollo de una actividad laboral identificable en el sistema productivo sin que ello constituya regulación del ejercicio profesional. 
los mecanismos de activación a seguir. Sin embargo, a partir de la aparición del Certificado de Profesionalidad en la Instrucción en Yoga, el panorama se modifica radicalmente, en tanto que se prevé como requisito indispensable para poder ejercer dicha profesión, especialmente por su normativa autonómica, en el caso catalán. Cabe recordar que los certificados de profesionalidad tienen carácter oficial y validez en todo el territorio nacional. Son expedidos por el SEPE y los órganos competentes de las Comunidades Autónomas. Este decreto establece los requisitos necesarios para poder acceder a dicha formación ${ }^{21}$. Así como las dos posibles vías para la obtención del certificado profesional de Instrucción en Yoga. La primera es superar todos los módulos formativos que integran el certificado de profesionalidad y que son un total de cuatro: Módulo 1 (Dominio de las técnicas específicas del yoga, 180 horas); Módulo 2 (Programación de actividades de instrucción en yoga, 120 horas); Módulo 3 (Metodología de la instrucción en sesiones de yoga, 160 horas) y Módulo 4 (Primeros Auxilios, 40 horas), a las que hay que sumar 120 horas de prácticas no laborables en un centro autorizado.

Tampoco los módulos formativos, tal y como se han planteado en la ley escapan a las críticas por parte del sector. Ello se debe a que la formación del yoga en nuestro país, aunque no tenía un carácter oficial sí que contaba, en la mayoría de los casos, con un reconocimiento y un aval por parte de las asociaciones y federaciones, tanto españolas ya citadas, como de ámbito europeo o internacional como son Yoga Alliance Internacional, European Yoga Federation por citar algunas de las más destacadas. Esta formación, ya consolidada, establece periodos de tiempo que oscilan entre 3 y 4 años de duración, según la escuela.

... Yo soy muy crítico con esto, porque yo creo que no tenía que haberse planteado como una formación profesional, es decir, lo que se ha hecho es una formación profesional pero a nivel de carpintero... eso es no entender nada. El programa tú lo ves y dices: ¡uy que programa! Pero ėen 6 meses de 8 a 2? Así, pues uno va a tener una formación como el que es carpintero, electricista... Pero no es lo mismo, nuestra formación, por ejemplo dura 4 años... (Sadhana)

Como se ha señalado, en el momento actual no se han activado los mecanismos necesarios para ofertar la formación de Instrucción en yoga en los Centros de Formación Profesional, pero al haberse aprobado los certificados de profesionalidad, el subsistema de formación para el empleo podrá, a partir de ahora, ser aplicado en las Comunidades Autónomas que a través de sus distintos servicios públicos de empleo, (ISOC en Cataluña, SERVEF en la Comunidad Valenciana, etc.) podrá ofertar el curso de Instrucción en Yoga. Para poder realizar dicha oferta, necesita a profesores de yoga

${ }^{21}$ Se deberán cumplir alguno de los siguientes requisitos: a) Estar en posesión del Título de Bachiller; b) Estar en posesión de un certificado de profesionalidad del mismo nivel del módulo o módulos formativos y/o del certificado de profesionalidad al que desea acceder; c) Estar en posesión de un certificado de profesionalidad de nivel 2 de la misma familia y área profesional para el nivel 3; d) Cumplir el requisito académico de acceso a los ciclos formativos de grado superior, o bien haber superado las correspondientes pruebas de acceso reguladas por las administraciones educativas; e) Tener superada la prueba de acceso a la universidad para mayores de 25 años y/o de 45 años; f) Tener los conocimientos formativos o profesionales suficientes que permitan cursar con aprovechamiento la formación. 
que impartan dichos cursos y éstos, han de cumplir con los requisitos de formación y experiencia profesional que exige el mencionado decreto ${ }^{22}$. Se requiere una titulación mínima de diplomado, siendo válida también la de licenciado, ingeniero, arquitecto o las actuales titulaciones universitarias de Grado. Para el módulo formativo de Primeros Auxilios se requiere la titulación específica en medicina o enfermería. Además, se requiere una experiencia profesional que varía en función del módulo formativo y que oscila entre uno y tres años en el ámbito de la unidad de competencia. También se requiere disponer del certificado de profesionalidad de formación ocupacional o formación equivalente en metodología didáctica de formación profesional para adultos. Así, por ejemplo, un antiguo diplomado o actualmente graduado en magisterio que posea un curso de formación como profesor de yoga y pueda acreditar la experiencia correspondiente podrá impartir clases en los módulos 1, 2 y 3, mientras que para el módulo 4 se requiere la titulación específica en el campo de la salud.

Son pues, los servicios públicos de empleo de cada comunidad autónoma los que buscado a las personas que se ajustan a este perfil a través de sus bases de datos y los que actualmente están confeccionando los materiales didácticos necesarios, así como las programaciones, etc. para ofertar dichos cursos, que al menos en un primer momento estaban dirigidos exclusivamente para desempleados. La oferta actual de centros donde se puede obtener el certificado de profesionalidad de Instrucción en yoga es muy limitada en el conjunto del territorio español. Aunque en la sede electrónica del SEPE, del Ministerio de Empleo y Seguridad Social aparecen un total de 31 centros que ofrecen dicha formación, 12 en Madrid, 5 en Andalucía, 3 en Comunidad Valenciana, 3 en el País Vasco, 2 en Navarra, 2 en Asturias, 2 en Murcia, 1 CastillaLeón y 1 en Cataluña, en realidad dicha oferta todavía no se ha materializado ${ }^{23}$.

El citado Real Decreto, 1076/2012, de 13 de julio, explicita las características que deben presentar los centros o escuelas (los requisitos mínimos de espacio, instalaciones y equipamiento establecidos en cada uno de los módulos formativos) que podrán ofrecer el certificado de profesionalidad de Instrucción de Yoga y que además, deberán cumplir con las prescripciones de los formadores. De esta forma se convierten en centros formativos de la red a la que se refiere la disposición adicional quinta de la Ley Orgánica 5/2002, de 19 de junio, de las Cualificaciones y de la Formación Profesional, previamente reconocido para ello por el Sistema Nacional de Empleo. También podrá realizarse dicha formación en la propia empresa cuando la misma dispusiere de las instalaciones y del personal adecuado a los efectos de la acreditación de la competencia o cualificación profesional, sin perjuicio de la necesidad, en su caso, de la realización de periodos de formación complementarios en los centros de la red mencionada. Se trata de centros que ofrecen otras formaciones de carácter deportivo y que forman parte de la lista de centros colaboradores con los servicios de empleo de que dispone cada comunidad autónoma. Así lo manifiesta un profesor de yoga consultado:

${ }^{22}$ De acuerdo con lo establecido en el artículo 13.3 del Real Decreto 34/2008, de 18 de enero, podrán ser contratados como expertos para impartir determinados módulos formativos, los profesionales cualificados con experiencia profesional en el ámbito de la unidad de competencia a la que está asociado el módulo.

${ }^{23}$ La Universidad Politécnica de Madrid es uno de los centros colaboradores que ya está ofreciendo dicha formación. 
... Está pensado yo creo, para las grandes empresas como CCC y estas que se dedican a la formación profesional que son a las que les van las subvenciones, a las que les va el dinero. Eso es una posible derivación de este problema... a los profesores también les piden una serie de requisitos que la mayoría no los van a tener para poder impartir clases... (Profesor de yoga)

Esto deja fuera, a la mayoría de centros de yoga en nuestro país, pues puede decirse que ninguno de ellos, cumple con dichas características (aunque en algunos casos, quizá puedan adaptarse). De ello se deriva el monumental enfado que se vive en el sector. Posteriormente, el Real Decreto 189/2013 de 15 de marzo, modifica el Real Decreto 34/2008, de 18 de Enero, por el cual se regulan los certificados de profesionalidad, introduciendo la posibilidad de impartir certificados de profesionalidad no financiados con fondos públicos desarrollados por empresas y centros de iniciativa privada. Lo que abre la puerta a que los centros y escuelas de yoga, siempre y cuando adapten sus instalaciones a lo que dice la ley, puedan ofertar esta formación de forma privada y reconocida a todos aquellos que lo deseen sin tener que ser necesariamente desempleados. Establece a su vez, medidas para favorecer la gestión eficaz de esta oferta para mejorar el seguimiento de la calidad en el desarrollo de la actividad formativa. Actualmente se prevé una Orden Ministerial que desarrolle dicho Real Decreto.

... esto es una carrera de obstáculos, porque tal y como estaba la ley, el certificado de profesionalidad solamente podía ofertarse a desempleados... (Profesora de Yoga)

La segunda vía es la de seguir los procedimientos establecidos para la evaluación y acreditación de las competencias profesionales adquiridas a través de la experiencia laboral o de vías no formales de formación. Ésta ha sido activada solamente en una comunidad autónoma, Cataluña, debido fundamentalmente, a que cuenta con la Llei 3/2008, del 23 d'abril, de l'exercici de les professions de l'esport, publicada en el DOGC y que tiene por objeto regular aspectos esenciales del ejercicio de determinadas profesiones del deporte, reconocer expresamente cuales son estas profesiones, asignarles las competencias asociadas, especificar las titulaciones o las acreditaciones, determinar las titulaciones necesarias para ejercerlas y atribuir a cada profesión el ámbito funcional específico que le corresponda.

Esta ley catalana incluye en el deporte todas las actividades físicas ejercidas en el deporte federado, el deporte escolar, el deporte universitario o en toda estructura o organización que promueva, organice o difunda este tipo de actividades, con independencia de la finalidad a que la actividad este destinada, ya sea de competición, iniciación, aprendizaje, tecnificación, salud, turismo, recreación, ocio o con finalidades análogas. En este sentido, afecta claramente a la profesión del yoga y es exclusivamente aplicable al ejercicio profesional en el ámbito territorial de Cataluña.

... En Cataluña lo que había hecho la gente es sacarse el título de monitor deportivo, son mil y pico de horas, son tres años. Pero cuando sale el certificado de Instructor en yoga cambia la cosa porque ahora lo que se requiere es tener este título para poder dar clases de yoga. Entonces la Conselleria impulso el proceso de Acreditación en Catalunya... (Profesor de Yoga)

De forma que hasta la aparición de los certificados de profesionalidad de Instrucción en Yoga, para ejercer como profesor de yoga en Cataluña se necesitaba la titu- 
lación como monitor deportivo que expedía la Escuela Catalana del deporte, sin embargo, ahora, tal como se publica en el blog de la Federació Catalana de Ioga:

Actualmente, el yoga al estar equiparado a una práctica deportiva y los profesionales del yoga a monitores deportivos, es la Escuela Catalana del Deporte quien puede cualificar a estos profesionales, pero resulta que la formación de tres años y la titulación de la Escuela Catalana del Deporte que permite ser monitor no da ni medio minuto de yoga, y por tanto no cumple las 550 horas que regula el Real Decreto 1076/2012, donde se fijan los módulos formativos y la duración de la formación en instructor de yoga. De esta forma, las escuelas privadas que vienen formando en yoga, y que son las que realmente saben de yoga, no solamente no pueden dar un título válido para ejercer la profesión de profesor de yoga porque aún no están acreditadas para ello sino que además, esta formación se convierte en una práctica delictiva, eso sí, solamente en Cataluña. (FCI)

Como hemos enunciado los certificados de profesionalidad son expedidos por el Servicio de Ocupación y Empresa de la Generalitat (SOC) que según regula el Real Decreto 189/2013, es a quién le corresponde la autorización, evaluación, seguimiento y control respecto a estas acciones formativas. Actualmente el SOC está trabajando para establecer los procedimientos y medidas necesarias para garantizar la adecuación y calidad de la formación impartida con financiación privada. El SOC, como organismo responsable de la autorización de esta formación y de la expedición de los certificados de profesionalidad en Cataluña, tiene la obligación de verificar que las acciones formativas se impartan de acuerdo con la normativa derivada del Real Decreto 34/2008, de 18 de Enero, así como de los reales decretos que regulan los certificados de profesionalidad impartidos.

En julio de 2013, se abrió la primera convocatoria de acreditación para obtener el certificado de profesionalidad de Instrucción en Yoga. A esta convocatoria podían presentarse todos aquellos que dispusieran de una formación cursada en la Asociación Española de Practicantes de Yoga (AEPY). Si dicha formación se había realizado hacia más de diez años, era necesario presentar la experiencia profesional de forma certificada. Es decir, especificando el puesto de trabajo como técnico o profesor de yoga durante al menos tres años con un mínimo de 2000 horas, en los últimos diez años. La persona autónoma podía presentar una declaración jurada ${ }^{24}$.

Cerrado el plazo de esta primera convocatoria se publicó una lista de admitidos y otra lista de espera de los aspirantes para proceder a una sucesión de entrevistas (informativas, de asesoramiento para la confección de dossiers) y finalmente una entrevista personal con cada uno de los aspirantes. Superada esta última entrevista, se entrega un certificado con el que debe hacerse la correspondiente solicitud al SOC que es el órgano competente para expedir el certificado de profesionalidad. Una vez finalizado el proceso de Acreditación es necesario apuntarse a la oficina de empleo, aunque sea como solicitud de mejora del puesto de trabajo. Esto sirve para que el ICQP transfiera los resultados al Servicio público de Empleo Estatal (SEPE).

Fueron numerosos los aspirantes que no obtuvieron dicha acreditación y que quedaron en lista de espera, de forma que la Resolución ENS/1236/2014 de 27 de mayo, abrió nuevamente una convocatoria pública para la evaluación y acreditación de com-

${ }^{24}$ En ambos casos hay que justificar el pago correspondiente de autónomos a la Seguridad Social. También se puede presentar un certificado de trabajo voluntario sin retribución. 
petencias profesionales adquiridas a través de la experiencia laboral o de vías no formales para el año 2014. Esta convocatoria estaba restringida únicamente a las personas que no obtuvieron plaza por falta de vacante en la convocatoria del año 2013 y dirigida, en primer lugar a las personas que quedaron excluidas por falta de vacante y a las personas que se les asignó pero quedaron en lista de espera. De esta forma, fueron muchos los que nuevamente quedaron excluidos de este proceso.

... Claro, este año seguimos igual, se ha abierto convocatoria pero no se ha abierto, solo para los que quedaron fuera en la anterior... así que no sabemos cuándo se abrirá nuevamente, suponemos que durante el 2015 pero no lo sabemos, ni tampoco si será en las mismas condiciones... ni nada de nada... (Profesora de Yoga)

Todo ello ha provocado fuertes discrepancias dentro del sector que han llegado a expresar a través de cartas abiertas en las distintas asociaciones y federaciones españolas. Así, tras los ataques recibidos, la Federación Española de Entidades Formadoras de Yoga (FEDEFY), responde al artículo publicado con fecha 28-04-14 con el título "La situación actual del Yoga" por la Federació Catalana de Ioga: "el contacto con la administración es para conseguir que la parte teórica del yoga, que no la espiritual ni las enseñanzas de los grandes Maestros, pudiera cualificarse oficialmente con la intención de evitar intrusismo y mala praxis en el mundo del yoga"; "decir que en la acusación que se hace a IYTA y AEPY de pertenencia masiva al tribunal de acreditación, con los "asesores y evaluadores", no hay presente más que un solo miembro de IYTA y un solo miembro de AEPY. Los demás son de otras escuelas porque se pasó correctamente esta información pública a la FEDEFY". Actualmente, junio 2015, se ha abierto una nueva convocatoria (ACREDITA'T) para 50 aspirantes.

En este proceso que se está desarrollando en Cataluña, siguen habiendo muchos interrogantes sin resolver, como el lugar donde podrán realizar las 120 horas de prácticas no laborables en un centro autorizado.

... Ahora la patata caliente esta en las prácticas, a ver como lo solucionan... se requieren 150 horas de prácticas en un centro que cumpla los requisitos, estos no van a poder absorber el número de alumnos, precisamente por las limitaciones que se les impone en los requisitos. (Profesor de yoga)

Prueba de todo ello es la moratoria, publicada en el DOGC de 21 de enero, que permite a los profesionales del deporte que no tengan titulación seguir prestando sus servicios hasta el 1 de enero de 2017 que finaliza el plazo otorgado por dicha moratoria.

Para el resto de comunidades autónomas el proceso de acreditación y habilitación abierto en Cataluña les ha creado un gran desconcierto. Puesto que, en primer lugar, desconocen los motivos por los que en otras comunidades autónomas no se esta realizando y cuando tratan de acceder a esta información y se dirigen a la administración autonómica tampoco la conoce y por lo tanto no puede ofrecerla.

... Aquí por ejemplo, hemos accedido a la consejería de deporte y bienestar, que estamos ahora en previos para poder homologar los cursos, a ver qué se puede hacer aquí en valencia porque parece que la información se queda en Barcelona... en conselleria nos han dicho que cuando sepamos algo que vallamos y se lo digamos, a ver qué hacemos, porque ellos no tienen ninguna información... (Gobinde) 
... Este proceso está siendo muy opaco, la información no llega, porque ya te digo cuando vas a hablar con los organismos oficiales, ahora aquí... aquí nadie sabe nada... (Profesora de yoga)

Y en segundo lugar, tampoco saben en qué posición quedan respecto a los catalanes. Puesto que ellos están obteniendo la titulación y el resto no.

... Pues sí, en el resto de España se percibe como que desde Cataluña se ha iniciado un proceso al cual el resto de España no ha sido invitado a participar y que ahora les afecta irremediablemente... (Profesor de yoga)

Así, están a la expectativa de ver cómo se va desarrollando el proceso en Cataluña y cuáles son las vías y los mecanismos que se activan en cada comunidad. Tal como manifiesta Mayte Criado, directora de la Escuela Internacional de Yoga y vicepresidenta de la FEDEFY en una entrevista publicada en la revista especializada Yoga en Red, "lo primero (y también lo más desesperante) es esperar a que cada Comunidad Autónoma anuncie oficialmente una convocatoria". Parece que en el caso de las comunidades autónomas de Madrid, País Vasco y Andalucía están en conversaciones para activar dicho proceso. En el siguiente fragmento de entrevista, sin embargo, destacan en el caso valenciano, la falta de predisposición por parte de la administración autonómica ante iniciativas que tengan que ver con el yoga

... Bueno, estamos a la expectativa de lo que va surgiendo y como se va desarrollando. De momento nosotros centrados en nuestro trabajo, en hacer nuestro trabajo, en formar a nuestra gente. Hace falta todo un conjunto de leyes para que se desarrolle y a ver como se hace también aquí en las comunidades, de momento aquí no hay nada... mira fueron a ofrecer estos cursos de yoga educa a la Conselleria para ver si se podía reconocer al igual que en Cataluña y dijeron que de yoga nada, no querían ni oír hablar de nada que tuviera yoga... (Sadhana)

En este contexto, los centros de yoga catalanes no han tenido más opción que ponerse manos a la obra con tal de ajustar su programa de formación a las horas que se especifican en la normativa, en poder ofrecer las infraestructuras necesarias, en disponer del profesorado habilitado para el ejercicio docente, etc. Cabe señalar que AEPY, está ajustando su temario y a través de sus escuelas de formación en "Instrucción en yoga" para emitir el certificado de estudios correspondiente al programa de cualificación. Por ello, ha programado un curso de primeros auxilios, para poderse presentar a la Acreditación (de momento en Catalunya).

En cambio, en el resto de comunidades autónomas, como por ejemplo en el caso valenciano, se observa como este proceso se ve todavía desde la distancia, aunque se entiende que cambiará radicalmente la formación de yoga en nuestro país. Porque "es muy importante haber elegido para formarse una escuela de yoga cuyo programa incluya los contenidos del Certificado de Instrucción en Yoga, ya que llegado el momento, deberá expedir a sus alumnos y ex alumnos un certificado detallado, firmado y sellado, con las horas exactas y los contenidos cursados durante la formación realizada" (Mayte Criado, Yoga en Red) y en caso contrario, estos alumnos no podrán obtener dicho certificado.

... Me llega la información y me entero... pero me mantengo muy al margen. Todo eso va a cambiar mucho en el futuro la formación, si se desarrolla... el panorama va a cam- 
biar mucho de la enseñanza, las condiciones que pone el Ministerio para poder enseñar el yoga aquí no las tenemos, se necesitan aulas, en fin una serie de condiciones que aquí no las tenemos, bueno, ya veremos qué pasa... (Sadhana)

Actualmente, los centros mantienen actitudes muy dispares que oscilan entre la indiferencia y continuar con lo que están haciendo sin querer involucrarse lo más mínimo en el proceso, hasta las que tratan de tener una posición activa e informarse para adaptarse a los requisitos requeridos en la ley y en medio de estas dos polaridades un continuum de posibilidades.

... Nosotros formamos a los profesores, tenemos esos estándares de horas, de calidad, creo que la tenemos, en el futuro, probablemente a lo mejor haya que darles un curso de primeros auxilios para homologar la titulación. Todo eso está por desarrollar, ya vendrá si viene... (Sadhana)

\section{REFLEXIONES FINALES}

En este artículo hemos realizado una brevísima introducción a los orígenes del yoga en la India, así como su extensión y difusión por el resto del mundo. Nos hemos detenido brevemente, en la penetración y en el proceso de implantación del yoga en el caso español, así, como su desarrollo. Actualmente, nos encontramos en un proceso de regulación de la profesionalización de la instrucción del yoga a raíz de los Reales Decretos publicados en los últimos años en materia de legislación. Hemos visto como se está realizando dicho proceso y las diferencias entre Cataluña y el resto de comunidades autónomas españolas, así como las fuertes discrepancias con la administración y entre el propio sector. Aunque son muchos los interrogantes abiertos con respecto al nuevo escenario que se configura en el mundo del yoga en el panorama español parece claro que la profesionalización del yoga es un hecho y que ello va a transformar en gran medida la configuración de centros y escuelas que habían funcionado hasta ahora. Aunque la práctica del yoga va mucho más allá de su profesionalización, los espacios que no se adapten a la nueva configuración jurídica tendrán que buscar otros caminos que les permita desarrollar otras dimensiones del yoga, la espiritual y la filosófica fundamentalmente, mucho menos popularizadas.

La aplicación de las normativas vigentes se encuentra en una fase inicial que todavía requiere de desarrollos jurídicos para su implantación, lo que no nos ha permitido realizar un análisis de la totalidad del proceso, ello no obsta para que resulte de gran interés conocer esta realidad y, ver como se está produciendo dicho proceso, puesto que puede ser el preludio de nuevas normativas en el ámbito de las medicinas alternativas y complementarias en nuestro país, que como ya hemos apuntado, su proliferación y su uso no dejan de crecer. En este sentido, queremos destacar que nos parece, adecuado y urgente llevar a cabo proyectos de investigación bien diseñados y con base población que permita conocer tanto la realidad de los profesionales como de los usuarios que utilizan las medicinas alternativas y complementarias para poder actuar en consecuencia y diseñar una normativa legislativa adecuada. Todo ello, acompañado de políticas que proporcionen seguridad al consumidor como una de las alternativas asistenciales y terapéuticas que tiene a su disposición (Perdi- 
guero 2004). De esta forma, no solamente podrían evitarse, tal y como hemos visto se han producido en el caso investigado, las fuertes discrepancias y críticas entre el sector del yoga y las diferentes instancias de gobierno, sino que dicho proceso se realizaría con un mayor conocimiento, previo diagnóstico, por parte de las instancias del gobierno que legislan y de la administración que aplica dichas leyes lo que con toda seguridad aumentaría las posibilidades de éxito.

\section{BIBLIOGRAFÍA CITADA}

Albert Rodrigo, María. 2014. "La proliferación de las Medicinas Alternativas y Complementarias. El ejemplo de la ciudad de Valencia". Revista de Antropología Experimental 14(13): 171-188.

Albert Rodrigo, María y Hernández Martí, Gil Manuel. 2014. “Los movimientos psico-espirituales en la modernidad globalizada". Revista AIBR 9(3): 273-296.

AEPY (Asociación Española de Practicante de Yoga). Disponible en: http://www.aepy.org/yoga/ la\%20tradicion.htm

Battles, Matthew. 2012. Lo intimo y lo sagrado. Conversaciones sobre la naturaleza profunda del ser bumano. Barcelona: Kairós.

Cornejo, Mónica y Blázquez, Maribel. 2013. "La convergencia de salud y espiritualidad en la sociedad postsecular. Las terapias alternativas y la constitución del ambiente holístico". Revista de Antropología Experimental 13: 11-30.

Díez de Velasco, Francisco. 2002. Introducción a la historia de las religiones. Madrid: UCM.

Eliade, Mircea. 1977. Yoga. Inmortalidad y libertad. Buenos Aires: Editorial La Pléyade.

Houtman, Dick y Aupers, Stef. 2007. "The Spiritual Turn and the Decline of Tradition: The Spread of Post-Christian Spirituality in 14 Western Countries, 1981-2000". Journal for the Scientific Study of Religion 46(3): 305-320.

Maffesoli, Michel. 2009. El reencantamiento del mundo. Una ética para nuestro tiempo. Buenos Aires: Dédalus Editores.

Mahadevan, Tellyavaram Mahadevan Ponnambalam. 1998. Invitación a la filosofía de la India. México: Fondo de Cultura Económico.

Perdiguero, Enrique 2004. "El fenómeno del pluralismo asistencial: una realidad por investigar». Gaceta Sanitaria 18: 140-145.

Prat, Joan (coord.). 2012. Els nous imaginaris culturals. Espiritualitats orientals, terapies naturals $i$ sabers esotèrics. Tarragona: URV.

Requena Segovia, Antonio. 1998. Una lluvia en el desierto. Málaga: PMA.

Roszak, Theodore. 1978. El nacimiento de una contracultura. Barcelona: Kairós.

Singleton, Mark. 2010. Yoga Body: the origins of modern posture practice. New York: Oxford University Press.

Yogananda, Paramahansa. 2008. Autobiografía de un Yogui. Los Angeles, California: Self-Realization Fellowship.

Fecha de recepción: 16 de febrero de 2015

Fecha de aprobación: 23 de junio de 2015 